\title{
Namen- und Sachregister (Auswahl)
}

\author{
Absalom 168, 169 \\ Achat siehe Steine \\ Adam 11, 113 \\ - neuer $90,113,118$ \\ - und Eva 89 \\ Adler siehe Tiere
}

Adressaten 118, 124, 169, 171

Affe siehe Tiere

Ägypten $11,17,23,25,35-37,39,43,45,46,57,59,75,86,93,122$, $124,125,144,153,155$

- Ägypter 22, 23, 34

- ägyptisch 22, 23, 29, 39, 56, 84, 97

Akademie 21

Alexander von Myndos 6

Alexandria, alexandrinisch 5, 20, 24, 35, 43, 45, 104, 105, 110, 112, $120,122-125,153,161,162,189,191-193$

Allegorese $68,71,72,74,84,85,87,88,94-98,114,115,117,125$, 126,133

Allegorie, allegorisch 6, 8, 11, 24, 55, 58, 64, 73, 74, 84-91, 94, 95, $97,105,118,123,124,126,133,134,155,162,163,168-171$, 178,180

Allegorisierung 139

Ambrosius 159-162, 166-168, 171

Ameise siehe Tiere

Ameisenlöwe siehe Tiere

Analogie 144

Anthropozentrismus 21, 31

Antichristus 146, siehe auch Teufel

Antilope siehe Tiere

Antiochenische Theologie 112, 113, 120

Antonius 154

Aphrodite 57

Apollinaris, Apollinarismus 111-113, 119, 121

Arche Noah 190, 191

Aristophanes von Byzanz 20

Aristoteles, aristotelisch 17-20, 22, 23, 30, 33, 39, 166

- Peripatetiker 30, 36

Askese, Asketen 55, 85, 123-125, 133, 135, 142, 154, 156, 160, 171, 172

Astarte 57,58

Atem 73, 127, 134, 139

Auferweckung siehe Christus, Auferstehung

Augustinus, augustinisch 85, 160, 162-166, 168-171

Autorität

- einer Person 6-8, 10, 11, 17, 19, 25, 26, 125, 126

- eines Textes 77, 161, 168

Biber siehe Tiere

Bilderbuchrollen 192

Bilderhandschrift 177

Bildung 20, 22, 36, 37, 63, 162, 171

- Bildungskanon, -programm 12, 162, 165

Blindheit 11

Blut 8, 10, 20, 21, 72-75, 103, 104, 134, 135, 144

- Bocksblut 5, 10

Bolos von Mendes 17, 21
Bongars, Jacques 183, 184

- Bongarsiana 177, 183, 184

Böse 10, 24, 42, 72, 73, 112, 133, 135, 144, 165, 168-170

- böser Geist 10, 29, 89, 170

Buße 8,74, 111

Castleton, Grafschaft Derbyshire 63

Celsus siehe Kelsos

Chorwerk 133, 135, 138, 140, 143

Christologie

- Engelchristologie 100, 102

- johanneische Christologie 141

- kosmische Christologie 104

- Logos-Sarx-Christologie 119, 121

- monophoysitische Christologie 119

- Pneuma-Sarx-Christologie 110

- Trennungschristologie 119,120

Christologie, christologisch $28,69,84,85,87,88,94,95,102,103$, $110,111,113,118-122,133-135,138,146,161$

Christus

- am Kreuz 8, 73-75, 94, 96, 100-104, 106-108, 115, 117-121, 135, 138, 139, 141-144, 192

- Auferstehung 11, 26, 28, 74, 85, 94, 96, 97, 100, 105, 107, 108, 110, $114,117-119,121,122,138,139,141,143-146,191,192$

- der Gesalbte 95, 114

- Engelgleichwerdung 96, 98, 100, 102-105, 108, 115, 116, 119, 123, 125

- Erlöser 18, 19, 42, 67, 68, 70, 73-75, 87, 93-103, 106, 108, 111, $114-118,122,141,142,145$

- Fleischwerdung 19, 68, 91, 94-109, 111-117, 119, 120, 122, 138 , $139,141,142,145,191$

- Geburt 17, 101, 114, 115, 119, 141

- Gottessohn 96, 97, 103, 109, 114, 115, 122, 123

- Gottheit, Gottsein ( $\theta$ cótns) 95-98, 108-122, 124, 191

- Göttlichkeit 96

- Himmelfahrt 97, 99, 100, 102, 105-109, 111, 112, 114-119, 121

- Höllenfahrt 28, 98, 100-102, 107-109, 115-117, 119, 123, 138, 145

- König 10, 57, 105, 106, 109, 115-117, 141

- Logos 91, 100-102, 104-106, 112, 113, 119, 133, 134, 139, 146, 190 , 191

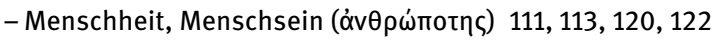

- Messiasgeheimnis 114

- Opfertod 74, 76, 103, 114, 133

- Taufe 58, 59, 110, 119

- Verhüllung 95, 96, 98, 109, 112-114, 116, 117, 191

- zur Rechten Gottes 96, 97, 105, 118-122

- Zweinaturenlehre 120

Chrysippos 21

Dämon 103, 104, 114, 151

Daniel, Pierre 183

David 42, 56, 72-76, 106, 126, 168, 169

- Haus Davids 68,141

- Prophet 108

descensus absconditus siehe Höllenfahrt

Diamant siehe Steine

Dionysios 6 
Doketismus, doketistisch 99-102, 115, 119

Drache siehe Tiere

Drei Jünglinge im Feuerofen 9, 150

\author{
Eidechse siehe Tiere \\ Einhorn siehe Tiere \\ Elefant siehe Tiere \\ Elias-Behang, Abegg Stiftung 192 \\ Emotion 27, 30, 190 \\ Engel 63, 68, 95-97, 99-103, 105-109, 111, 114-118, 122, 127 \\ Enthaltsamkeit 12, 59, 89 \\ Erlösung 70, 74, 126, 142, 144 \\ Esel siehe Tiere \\ Eule siehe Tiere \\ Eusebius von Caesarea 163, 164 \\ Evangelium 10, 73, 103 \\ ewiges Leben $8,74,135,143$
}

Fabeltiere, Fabelwesen 7, 55, 68, 71, 83, 140, 153, siehe Mischwesen

Falke siehe Tiere

Fasten 12, 28, 36, 142

Fisch siehe Tiere

Fischotter siehe Tiere

Fleury 182, 183

Fortpflanzung 19, 55, 89, 90, 166, 167, 169

Frosch siehe Tiere

Frowin, Abt 135

Fuchs siehe Tiere

Gazelle siehe Tiere

Geier siehe Tiere

Gerechtigkeit 11, 24, 31, 33, 87

Gjeilo, Ola 133, 135, 138, 140, 142, 143, 145, 146

Glaube 11, 12, 25, 85, 91, 142, 165

Gnosis, Gnostiker, gnostisch, gnostizierend 93, 95, 98-102, 104, $105,115,119,122,123,125,149$

Gotteserkenntnis 25, 86

Gotthelf, Jeremias 127

Gottvater 95, 97, 100, 113, 118-122, 139, 190, 191

Graviseth, Jakob 184

Graviseth, Reinhard 184

Gregor der Große 166, 169-171, 182, 183

Hades 119, 120, 145

Hagen, Hermann 184

Handschriften

- Artemidorus Papyrus (Fondazione per l'Arte della Compagnia di San Paolo, Turin) 45, 46

- Charta Allaciana (Biblioteca Vallicelliana, Rom)

- 216 (CXLI) 10

- Climax lacobi (slawisch-orthodox)

$-4.4695$

- Codex 10074 (Bibliothèque Royale, Brüssel) 177, 178

- Codex 225 (Burgerbibliothek Bern) 183, 186

- Codex 231 (Burgerbibliothek Bern) 184

- Codex 233 (Burgerbibliothek Bern) 177-179, 182-184, 186, 187

- Codex 318 (Burgerbibliothek Bern) siehe Physiologus Bernensis

- Codex 397 (Pierpont Morgan Library, New York) 5

- Codex 462 (Burgerbibliothek Bern) 184

- Codex 611 (Burgerbibliothek Bern) 177-179, 181-184, 187
- Codex 847.9 (Burgerbibliothek Bern) 183

- Codex Gudianus latinus 148 (Herzog August Bibliothek, Wolfenbüttel) 178

- Codex Lipsiensis Universitatis 35 (Universität, Leipzig) 73

- Codex Manichaicus Coloniensis (Papyrussammlung, Universität Köln)

$-2.10101$

$-38.2-3101$

$-41.11101$

- Codex Monacensis latinus 14388 (Bayrische Staatsbibliothek, München) 178

- Codex Monacensis latinus 19417 (Bayrische Staatsbibliothek, München) 178

- Codex Mosquensis Graecus 432 (Synodalbibliothek, Moskau) 178

- Codex Ms. 313 (Bibliothèque municipale, Orléans) 183

- Codex Oxoniensis Baroccainus graecus (Bodleian Library, Oxford) 73

- Codex Par. lat. 10756 (Bibliothèque Nationale, Paris) 181

- Codex Vaticanus graecus 695 (Biblioteca Apostolica Vaticana Rom) 73

- Codex Par. Gr. 1197, Theodoros Studites (Bibliothèque nationale de France, Paris) 151

- Codex Par. Gr. 1468v (Bibliothèque nationale de France, Paris) 151

- Cotton Otho B4 (Cotton Genesis, British Library London) 189-191

- Ebo-Evangeliar (Ms. 1, Bibliothèque Municipale Ebernay) 190, 192

- Evangeliar (Codex Par. lat. 17968, Bibliothèque Nationale, Paris) 190

- Evangeliar (M. 728, Pierpont Morgan Library, New York) 190

- Evangeliar (Ms. 7, Reims) 190

- Josua-Rolle (Cod. Vat. Palat. gr. 431, Biblioteca Apostolica Vaticana Rom) 190

- Minuskel GA 537 (University of Michigan) 83

- Papyrus Berol. (inv. 7999, Ägyptisches Museum und Papyrussammlung, Staatliche Museen zu Berlin) 43, 44

- Papyrus PSI XVI 1577 (inv. 295, Istituto Papirologico Girolamo Vitelli, Florenz) 39-43

- Physiologus Bernensis (Codex 318, Burgerbibliothek Bern) 64, 177-180, 183, 184, 189-192

- Physiologus von Smyrna (zerstört, nur Fotos erhalten) 192

- Prudentius (Codex 264, Burgerbibliothek Bern) 189, 192

- Quedlinburger Itala (Cod. theol. lat. 285, Staatsbibliothek Berlin (4 Blätter) und Stiftskirche St. Servatius in Quedlinburg (1Blatt)) 190

- Rabbula-Codex (cod. Plut. 1.56, Biblioteca Medicea Laurenziana, Florenz)

- fol. 13v 122

- Utrecht Psalter (Ms. 32, Universitätsbibliothek Utrecht) 190, 191

- Vergilius Romanus (Vat. lat. 3867, Biblioteca Apostolica Vaticana Rom) 191

- Vergilius Vaticanus (Vat. lat. 3225, Biblioteca Apostolica Vaticana Rom) 189,190

-f.9r 192

- Wiener Genesis (cod. theol. gr. 31, Österreichische Nationalbibliothek, Wien) 192

Hathor 61

Heilige Schrift siehe Bibel

Heiliger Geist 85, 105, 106, 108

Heilsgeschichte $23,77,89,101,138,146,190,192$

Hellenismus, hellenistisch, hellenistisches Judentum 6, 20, 22, 23 , 58,59 
Heraklit 20

Hermeneutik 55, 112, 123, 125, 134, 135, 138, 139, 160, 162, 169

Hieronymus $159,161,182,183$

Himmel 95, 97, 99-109, 112-114, 117-121

- Tore 63, 96, 99, 102, 105-109, 116-118

Hinkmar, Erzbischof 190

Hirsch siehe Tiere

Hirt 55, 56, 113

Homer 6, 8

Hortin, Samuel

- Clavis bibliothecae Bongarsianae (Cod. A 5, Burgerbibliothek Bern) 184

Hund siehe Tiere

Hymnus 20, 121, 133, 135, 138, 140-144, 146, 192

Hyäne siehe Tiere

Ibis siehe Tiere

Ichneumon siehe Tiere

Igel siehe Tiere

Instinkt 21, 31

Intertextualität 85, 86, 88-91, 142

Ischtar 56, 57, 60, 62

Isidor 180, 182, 183

Israel 55-58, 69, 96, 143, 146

Jäger $19,22,46,70,89,94,97,115-117,136,139,140,151$

Jakob 126, 190-192

Jeremia 126

Jesus siehe Christus

Johannes der Täufer 42, 113, 126, 166

Juba II. von Mauretanien 6

Juden, Judentum 18, 24, 25, 63, 87, 88, 95, 96, 109

Justin der Märtyrer 106

Karfunkelstein siehe Steine

karolingische Renaissance 192, 193

Käuzchen siehe Tiere

Kelsos 12

Krähe siehe Tiere

Krokodil siehe Tiere

Kuckuck siehe Tiere

Kunst

- Buchmalerei 68, 189, 191-193

- Katachombenmalerei 9

-Wandmalerei 191

Lamm siehe Tiere

Lazarus 145, 146

liber creaturae, liber naturae $6,83,162,165,168,169$

Licht 11, 18, 87, 97, 144, 145, 169, 190

Liebe

- Christi $18,19,87,88$

- Elternliebe 21, 27, 33, 45, 74, 75

- Gattenliebe 33, 35, 57, 58

Logos 91, 97, 141, 191

Lorrain, Claude 190

Lösegeld 112-114

Löwe siehe Tiere

Lust 151, 155
Maat 60

Mächte $68,70,95-109,112-117,119,121,124$

Magnetstein siehe Steine

Malerei siehe Kunst

Maria 68, 70, 95-98, 101, 141, 192

Mensch 9, 21, 31-36, 85, 86, 89-91, 95, 120, 139, 144, 192

Metamorphose 7, 35, 36, 100, 102

Metapher 55, 58, 59, 113, 121, 126, 143, 162, 165-169

Mirabilienliteratur 20, 21, 29, 134, 153

Mischwesen 6-8, 12, 142, 192

Mosaik 140, 189, 191

Mose 23-25, 103, 142, 143, 150

Muschel siehe Tiere

Musik siehe Chorwerk, siehe Gjeilo, siehe Hymnus

Nachtrabe siehe Tiere

Natur

- deutung 84, 87, 88, 90, 91, 138, 172

- kunde 12, 17, 19, 59, 97, 159-165, 171, 172

- theologie 83

Neugierde 12, 163, 164, 172, 192

Neuschöpfung 71, 139, 145, 146

Offenbarung 12, 105, 126, 127

Ontologie 19, 95

Oppian 6

Orakel 94

Origenes 100,102-111, 114, 117, 119, 151, 155

- antiorigenistisch 104

Palimpsest 181

Panther siehe Tiere

Paradies 89, 119, 120, 126, 142, 143

Paradoxographie siehe Mirabilienliteratur

Paulinus von Nola 161

Paulus 22, 42, 150

Pelikan siehe Tiere

Perle siehe Steine

Pétau, Paul 183

Pferd siehe Tiere

Philon von Alexandrien 6, 24, 25, 27, 105

Physiologus siehe Stellenregister

Physiologus Bernensis siehe Handschriften

Phönix siehe Tiere

Platon, Platoniker, platonisch 23, 24, 31, 34, 36

Plinius der Ältere 27, 29

Plutarch 22-25, 27-29, 31-37

poetisch $73,85,86,88,89,146,168$

Poseidonios 21

Prudentius 166, 168, 169, 171

Pyrrhon, Pyrrhoneer 31, 35, 36

Pythagoras, Pythagoreer, pythagoreisch 21-23, 31, 155

Qedeschet 56,61

Regenpfeifer siehe Tiere

Reihervogel siehe Tiere

Reims 180, 183, 190, 192, 193

Reinheit $65,67,75,77$

res - signa 162,163 
Rhinoceros siehe Tiere

Rom 153, 164, 192

Rufin von Aquileia 103, 160, 161

Sachmet 56, 61

Salamander siehe Tiere

San Marco, Venedig 189, 191

Santa Maria Antiqua, Rom 190

Santa Maria Maggiore, Rom 189

Satan siehe Teufel

Schlange siehe Tiere

Schwalbe siehe Tiere

Schönheit 86, 87

- der Geschöpfe 87, 90

- der Natur 86

- der Sterne 87

- der Tiere 20

- des Göttlichen 23, 105

- des Menschen 20

Schöpfung $8,12,30,65,75,84,96,101,119,126,133,134,139$, $140,146,159,160,165,172,190$

- Schöpfer 8, 75, 87, 90, 190

- Schöpfungstheologie 126,139

Seehund siehe Tiere

Seele

- Christi 119-121

- der Pflanzen 30, 34

- der Tiere 30, 33, 36

- des Menschen 30, 63, 71, 72, 101, 133, 134, 167, 168

- körperlose Wesen 33

- Seelenwanderung 7

Sextus Empiricus 27, 31, 32, 36

Simon Magus 93, 98, 125

Sintflut 57

Skarabäus siehe Tiere

Speisegesetze 23-26, 67, 75

Steine 7,11, 33, 64

- Achat 7, 21, 85, 143

- Diamant 5-7, 9, 10, 21, 40, 42, 125

- Karfunkelstein 6

- Magnetstein 7, 21, 154

- Perle 7, 85, 109, 143, 152

Stern $19,30,85$

- Gestirne 33, 86

- Morgenstern 34

- Wunderstern 101

Stoa, Stoiker, stoisch 21, 22, 24, 30-34, 36, 126

Sünde $18,19,87,88,135,136,144,168,169$

- Erbsünde 169

- Sündenfall 7, 89, 114, 142, 143

- Sünder 111, 168

- Todsünde 168

Taube siehe Tiere

Teufel $10,29,63,72,73,88,90,97,98,101,109,112-114,117,123$, $134,135,145,146,151,170,171$

Thekla 150

Theophilos von Alexandria 104

Theophrast 6
Tiere

- Adler 7, 8, 18, 58, 66, 67, 76, 86, 90, 126, 152

- Affe 7, 65, 66, 76, 88, 90

- Ameise 7, 31, 65-67, 70, 71, 76, 85, 90, 192

- Ameisenlöwe 7, 64-67, 70, 71, 76, 77, 153

- Antilope 7, 65, 66, 76

- Biber 7, 22, 46, 65, 66, 76, 124

- Drache 45, 97, 142

- Eidechse 10, 11, 18, 66, 76, 192

- Einhorn 5, 7, 17, 64-72, 74, 76, 77, 90, 97, 126, 133, 136, 138-142, $146,153,192$

- Eisvogel 7, 33-36

-Elefant 7, 19, 20, 33, 34, 45, 46, 65, 66, 76, 89, 90, 118, 126, 155, 192

- Esel 57, 58

- Eule 154

- Falke 23

- Fisch 6, 7, 25, 31, 34, 46, 65, 76, 86, 112, 149

- Fischotter 7, 65, 66, 76, 98, 119, 138, 145, 146

- Frosch 7, 65, 66, 76, 124

- Fuchs 7, 21, 65-67, 76, 90

- Gazelle 40, 42, 46, 65, 66, 76, 125, 143

- Geier 7, 58, 66, 67, 76, 109, 121

- Hirsch 63, 65-67, 70, 72, 73, 76, 77, 133-135, 142, 191

- Hund 31, 33, 55, 95

- Hyäne 7, 19, 20, 25, 65-67, 76, 155

- Ibis $11,12,22,25,36,66,67,76,179$

- Ichneumon 7, 33, 65-67, 76, 90, 97, 98, 109, 114, 117, 118, 142

- Igel 7, 28, 29, 55, 65, 66, 76

- Käuzchen 7, 8, 18, 66, 67, 75, 76, 87, 88, 90, 118, 191

- Krähe 7, 33, 66, 67, 76, 155

- Krokodil 7, 22, 33, 34, 66, 98, 119, 136, 140, 145

- Kuckuck 7,151

- Lamm 66, 76, 96, 136, 138, 140, 143, 144

- Löwe 7, 22, 56, 58, 60-62, 65-67, 71, 72, 76, 86, 90, 94-97, 109, $115-119,121,122,124,125,134,136,138-141,143-146,161$, 190-192

- Muschel 21, 85

- Nachtrabe 7,18-20, 25, 66, 75, 76, 87, 191

- Panther 7, 28, 31, 36, 45, 46, 56, 65-67, 76, 143, 153, 179

- Pelikan 7, 8, 21, 45, 46, 55, 66, 67, 74-77, 87, 90, 126, 136, 138, 144,153

- Pelikan (4) 66

- Pferd 55, 57, 58, 192

-Phönix 5, 7, 55, 66, 67, 74, 76, 90, 138, 140, 144, 153

- Rebhuhn 7, 66, 67, 76, 151, 152, 155, 156

- Regenpfeifer 66, 67, 76, 91, 109, 142, 192

- Reihervogel 66,67

- Rhinoceros 69

- Salamander 5, 7, 9, 66, 76, 164

-Schlange 7, 11, 22, 23, 34, 36, 45, 46, 55, 61, 66, 67, 72, 73, 77, 89, $126,133-136,138,140,142-146,152,160,166-171,192,193$

- Natter 21, 63, 66, 76, 109, 142, 166-168, 170

- Otter 63

- Schlangengift 72, 136, 142, 167, 168, 170

- Viper 63, 142, 168-170, 192

- Schwalbe 66

- Seehund 150

- Skarabäus 22, 23

- Taube 7, 22, 55, 57-59, 66, 67, 76, 142, 152, 159 
- Tiger 28

- Ungetüm „Säge“ 65, 66, 76

-Walfisch 21, 65, 66, 76

- Wiedehopf 7, 22, 66, 76, 90, 154

-Wiesel 5, 7, 19, 22-25, 65-67, 76, 90

- Wildesel 10, 57-59, 65-68, 76, 88, 90, 140, 149, 151, 152, 154

Tierkult 10, 22, 23, 35-37

Tiersymbolik 17, 20, 22-24, 26, 55, 58, 86, 94, 133, 138, 160

Tiger siehe Tiere

Tod, tot $8,18,34,35,58,74,75,87,108,134,136,139,140$, 142-146, 167-169, 171

Triumphsäulen, Rom 192

Tugend 21, 30, 31, 33, 167

Typologie 26, 84, 85, 126, 169

Ungetüm „Säge“ siehe Tiere

Unreinheit, unrein $24,25,58,65,67,75,77,87$

Vegetarismus 21, 31

Venus 57
Vergänglichkeit 33,143

Vernunft 21, 22, 27, 31-34, 104, 164

- vernunftlos 31-33

Versuchung 12, 113, 124, 135, 169-171

Vetus Latina 111, 178

Wahrheit 32, 73, 83, 85, 91, 100, 111

Walfisch siehe Tiere

Wasser 8, 33, 58, 62, 72-75, 89, 90, 133-135, 144

Weisheit 27, 36, 55, 58, 100, 101, 125, 135, 143, 163

Wellmann, Max 17, 23, 153

Wiedehopf siehe Tiere

Wiesel siehe Tiere

Wild, Marquard 184

- Katalog (Cod. A 4, Burgerbibliothek Bern) 184

Wildesel siehe Tiere

Wunder 9, 12, 107, 110, 111, 151, 161, 164, 165, 171

Wüste $8,22,29,74-76,142,154$

Xenokrates von Ephesos 6,10 
